\title{
Optimally shaping the stationary distribution of a constrained discrete time stochastic linear system via disturbance compensation
}

\author{
Alessandro Falsone, Luca Deori, Daniele Ioli, Simone Garatti, Maria Prandini
}

\begin{abstract}
We consider the problem of shaping the distribution of the state of a discrete time linear system subject to a stationary disturbance so as to optimize a performance index while satisfying probabilistic constraints involving control input and state. The state is not available and a disturbance compensator is designed via a one-shot off-line computational approach to optimally operate the system in stationary conditions. The resulting control strategy is easy to implement, able to handle probabilistic constraints and guarantees optimality in the long run. Moreover, it does not require the on-line (re)computation of the control parameters. Interestingly, problems where the disturbance is cyclostationary and/or the control is periodic can be embedded in our formulation. This is the case of the numerical example presented in the paper, where the proposed methodology is applied to stochastic periodic control of a battery for peak shaving of the solar energy produced by a photovoltaic panel installation.
\end{abstract}

\section{INTRODUCTION}

We consider a discrete time linear time invariant system affected by a stationary additive disturbance. Our goal is to design a control strategy so as to optimize the system performance and satisfy some probabilistic constraint when the system is operating in stationary conditions. This involves characterizing the stationary state process and optimally shaping its distribution.

The problem formulation resembles that of minimum variance (MV), originally introduced in [1], and generalized minimum variance approaches (GMV), [2], [3], [4], [5], [6], [7], [8]. In GMV, actuation constraints are typically accounted for through the introduction of suitable weights in the cost function, [9]. The problem addressed in this paper is more general in that other performance indices than variance can be considered and joint state and input constraints are directly accounted for, via a chance constrained problem formulation, with constraints imposed in probability.

In this paper, we are concerned with the case when the state is not available for control input design, while instead disturbance measurements are. A disturbance compensator is thus designed to shape the stationary distribution. The compensator parameters are computed once, off-line, and then the compensator is implemented on-line, without requiring any (re)computation of its parameters. Once the controlled system has reached steady-state conditions, the resulting stationary state process is guaranteed by construction to satisfy the probabilistic constraints and to optimize the performance.

Research was supported by the European Commission under the project UnCoVerCPS, grant number 643921.

Alessandro Falsone is an IEEE Student Member.

The authors are with the Dipartimento di Elettronica Informazione e Bioingegneria, Politecnico di Milano, Piazza Leonardo da Vinci 32, 20133 Milano, Italy, e-mail: \{alessandro.falsone, luca.deori, daniele.ioli, simone.garatti, maria.prandini\}epolimi.it
The off-line computation of the compensator parameters is a hard problem, in general, due to the presence of probabilistic constraints. In our set-up, the problem is even harder since the probability appearing in the constraints refers to the whole disturbance process. In this case, analytic methods for approximating the probabilistic constraints, [10], [11], [12], as well as randomized methods, [13], [14], [15], [16], are not directly applicable. The main theoretical contribution of this paper is the extension of the scenario theory results on chance constraints feasibility to the proposed setting, by approximating the stationary state distribution and accounting for the introduced approximation error via constraint tightening.

The considered control set-up is particularly appealing for those applications where the state is hardly available, because of, e.g., the lack of suitable sensors. We actually developed this work motivated by an energy management application and, in particular, the cooling of buildings in a microgrid with common resources like thermal storages, [17], [18]. To model the inertia of the building, which acts as a passive storage, a high dimensional model is introduced where the building walls are sliced in layers, and the temperatures of all layers are taken as state variables. It is clear then, that in this problem the state is not available, while measurements of the disturbances as given by outside temperature and solar irradiation are easily obtained.

It is worth noticing that outside temperature and solar irradiation are typically cyclostationary processes, not stationary as required by our approach. A stationary set-up can however be recovered by unrolling the system dynamics and referring to a time interval length of one-day period as time step for the state evolution. This finally leads to a stochastic periodic control solution, as shown in the numerical example presented in the paper, where a simpler application to peak shaving of solar energy production using a battery is addressed.

The rest of the paper is organized as follows. We first provide some notations. The problem addressed is precisely formulated in Section II. Our scenario-based solution and the main result on chance constraints feasibility are stated in Section III. A numerical example is illustrated in Section IV. Finally, some concluding remarks are drawn in Section V.

Notation: A discrete time process $\left\{v_{k}, k \in \mathbb{Z}\right\}$, is also denoted as $\boldsymbol{v}$, while its probability distribution is $\mathbb{P}_{\boldsymbol{v}}$. Correspondingly, the expected value with respect to $\mathbb{P}_{\boldsymbol{v}}$ is denoted by $\mathbb{E}_{\boldsymbol{v}}[\cdot] . I$ denotes the identity matrix, $S=$ $\operatorname{diag}\left(s_{1}, \ldots, s_{m}\right)$ the diagonal matrix built from the scalars $s_{1}, \ldots, s_{m}$, and $J=\operatorname{blkdiag}\left(J_{1}, \ldots, J_{m}\right)$ the block diagonal matrix built from the square matrices $J_{1}, \ldots, J_{m}$. We denote by $\rho_{X}=\max \{|\lambda|: \operatorname{det}(\lambda I-X)=0\}$ the spectral radius of a square matrix $X$, and by $\|\cdot\|_{p}$ the matrix norm 
induced by the $p$-norm for vectors. Finally, $\stackrel{L^{1}}{\rightarrow}, \stackrel{L^{2}}{\rightarrow}$, and $\stackrel{P}{\rightarrow}$ denote mean absolute value convergence, mean square convergence, and convergence in probability.

\section{Problem FORMUlation}

Consider a linear system where the state $x_{k} \in \mathbb{R}^{n_{x}}$ evolves according to the discrete-time equation

$$
x_{k+1}=A x_{k}+B u_{k}+W d_{k},
$$

where $u_{k} \in \mathbb{R}^{n_{u}}$ and $d_{k} \in \mathbb{R}^{n_{d}}$ represent the control input and an additive stochastic disturbance, respectively. $A, B$, $W$ are matrices of appropriate dimensions.

Throughout, we make the following assumptions.

Assumption 1 (Asymptotic stability): All the eigenvalues of matrix $A$ are strictly contained in the unit circle centered in the origin of the complex plane, i.e., $\rho_{A}<1$.

Assumption 2 (Disturbance): The stochastic process $\boldsymbol{d}$ is strictly stationary with zero mean and well-defined and known second order moment.

The zero mean assumption is without loss of generality, because if this is not the case, we can introduce

$$
\bar{x}_{k+1}=A \bar{x}_{k}+W \bar{d},
$$

where $\bar{d}=\mathbb{E}_{\boldsymbol{d}}\left[d_{k}\right]$, and reformulate the problem in terms of $\Delta x_{k}=x_{k+1}-\bar{x}_{k}$, which evolves according to

$$
\Delta x_{k+1}=A \Delta x_{k}+B u_{k}+W \Delta d_{k},
$$

where $\Delta d_{k}=d_{k}-\bar{d}$ is zero mean.

Assumption 3 (Information structure): At time $k \in \mathbb{Z}$, the value taken by $d_{k}$ is available for compensation purposes. The state process $\boldsymbol{x}$, instead, is not available.

Let the control input $u_{k}$ be parameterized as the following static function of $d_{k}$

$$
u_{k}=\gamma+\vartheta d_{k}
$$

where $\gamma$ and $\vartheta$ are the controller parameters taking values in the convex and compact sets $\Gamma \subset \mathbb{R}^{n_{u}}$ and $\Theta \subset \mathbb{R}^{n_{u} \times n_{d}}$, respectively. By plugging the disturbance compensator (2) into (1), we obtain

$$
x_{k+1}=A x_{k}+B \gamma+(B \vartheta+W) d_{k} .
$$

Given an integer $M \in \mathbb{N}$, let us define process $\boldsymbol{x}_{\boldsymbol{M}}=$ $\left\{x_{k, M}, k \in \mathbb{Z}\right\}$ where

$$
x_{k, M}=(I-A)^{-1} B \gamma+\sum_{s=0}^{M-1} A^{s}(B \vartheta+W) d_{k-1-s} .
$$

Under Assumptions 1 and 2, by [19, Theorem 1.4 (p. 80)], $\forall k$ there exists a $x_{k, \infty}$ such that $x_{k, M} \stackrel{L^{2}}{\rightarrow} x_{k, \infty}$ (i.e. $x_{k, M}$ is mean-square convergent to $x_{k, \infty}$ ) as $M \rightarrow \infty$. Moreover, $x_{k, \infty}$ is a measurable function of process $\left\{\ldots, d_{k-2}, d_{k-1}\right\}$, so that the probability distribution of $x_{k, \infty}$ is induced from that of $\left\{\ldots, d_{k-2}, d_{k-1}\right\}$, and the process $\boldsymbol{x}_{\infty}=$ $\left\{x_{k, \infty}, k \in \mathbb{Z}\right\}$ satisfies (3) and is strictly stationary with well-defined finite first and second order moments.

Our goal is to shape the distribution of the stationary state $x_{k, \infty}$ by choosing the compensator parameters $\gamma$ and $\vartheta$ so as to optimize some performance criterion while satisfying state and input constraints. In this respect, it is worth noticing that, although the disturbance $d_{k}$ is available (see Assumption 3), it is typically not possible to cancel out its contribution on the state dynamics (1) by means of the term $B u_{k}$, also because $u_{k}$ may be subject to constraints.

Here, we focus on the following formulation of the problem

$$
\begin{array}{rl}
\min _{\gamma \in \Gamma, \vartheta \in \Theta, h} & h \\
\text { subject to: } & \mathbb{P}_{\boldsymbol{d}_{\boldsymbol{k}}\{}\left\{\ell\left(x_{k, \infty}, \gamma+\vartheta d_{k}, d_{k}\right) \leq h\right. \\
& \left.\wedge f\left(x_{k, \infty}, \gamma+\vartheta d_{k}\right) \leq 0\right\} \geq 1-\varepsilon .
\end{array}
$$

where $\varepsilon \in(0,1)$ is a user-chosen probability level and $\mathbb{P}_{\boldsymbol{d}_{\boldsymbol{k}}}$ is the probability distribution of process $\boldsymbol{d}_{\boldsymbol{k}}=\left\{\ldots, d_{k-1}, d_{k}\right\}$. Function $\ell(x, u, d) \in \mathbb{R}$ associates a cost to the state and control input pair $(x, u)$ when the disturbance value is $d$, whereas function $f(x, u) \in \mathbb{R}$ defines a joint constraint on $(x, u)$. The fact that $f(\cdot)$ is a single constraint function is without loss of generality because if multiple constraint functions $f_{1}(\cdot), \ldots, f_{m}(\cdot)$ are present, then, we can redefine $f(\cdot)$ as their maximum. In (5), we are then minimizing the cost while satisfying the joint state and input constraint for all disturbance realizations except for a set of probability at most $\varepsilon$.

Both functions $\ell(x, u, d)$ and $f(x, u)$ are evaluated in stationary conditions, i.e., with $x$ set equal to the stationary state $x_{k, \infty}$ and with $u$ given by the disturbance compensator in (2). By the joint stationarity of processes $\boldsymbol{x}_{\infty}$ and $\boldsymbol{d}$, the solution to (5) is optimal and satisfies the probabilistic constraint in (5) for all time instants $k \in \mathbb{Z}$. This is particularly appealing because the control law can be computed once (off-line), and, then, be applied at each time step without solving any further on-line optimization problem. Optimality and feasibility, however, are guaranteed for all $k$ only when the system is operating in stationary conditions. In practice, thanks to Assumption 1, stationarity is always reached in the long run, with a convergence rate that depends on $\rho_{A}$.

The following assumptions on the cost function $\ell(\cdot)$ and on the constraint function $f(\cdot)$ are required to develop our resolution approach to (5).

Assumption 4 (Convexity): The cost function $\ell(x, u, d)$ and the constraint function $f(x, u)$ are convex with respect to $(x, u) \in \mathbb{R}^{n_{x} \times n_{u}}$.

Assumption 5 (Lipschitz continuity): The cost function $\ell(x, u, d)$ and the constraint function $f(x, u)$ are Lipschitz continuous in $x \in \mathbb{R}^{n_{x}}$, with Lipschitz constant $L$, for any $(u, d) \in \mathbb{R}^{n_{u} \times n_{d}}$.

Remark 1 (average cost): It is perhaps worth mentioning that the results of the paper have more general validity than for the problem considered in (5). In particular, one can also minimize the average cost $J(\gamma, \vartheta)=$ $\mathbb{E}_{\boldsymbol{d}_{\boldsymbol{k}}}\left[\ell\left(x_{k, \infty}, \gamma+\vartheta d_{k}, d_{k}\right)\right]$ leading to

$$
\begin{array}{ll}
\min _{\gamma \in \Gamma, \vartheta \in \Theta} & J(\gamma, \vartheta) \\
\text { subject to: } & \mathbb{P}_{\boldsymbol{d}_{\boldsymbol{k}}}\left\{f\left(x_{k, \infty}, \gamma+\vartheta d_{k}\right) \leq 0\right\} \geq 1-\varepsilon .
\end{array}
$$

In this case, the convexity assumption is required for $J(\cdot)$ and $f(\cdot)$, while the Lipschitz continuity assumption for $f(\cdot)$ only. The theory developed next would still apply to this set-up with minor modifications. 
Remark 2 (Stochastic periodic control): Optimal stochastic periodic control can be embedded in our framework by adopting the so-called lifting transformation, i.e., by unrolling the original system dynamics over a time window of length equal to the periodicity $T$ and referring to the system dynamics sampled at a slowest rate. The stationarity of $\boldsymbol{x}_{\infty}$ then maps back into the cyclostationarity of the original state process with period $T$. This can be particularly useful, for instance, when dealing with a system affected by a cyclostationary disturbance and/or a control problem with cost and input/state constraints that refer to some finite time horizon. An instance of this kind of problem will be described in Section IV.

\section{PROPOSED SOLUTION AND MAIN RESUlT}

Chance constrained problems like (5) are generally challenging to solve, because of the probabilistic constraint which can make the optimization problem non convex even under Assumption 4 on the convexity of both $\ell(\cdot)$ and $f(\cdot),[20]$, [21]. A further difficulty that we need to face in our set-up is that $\mathbb{P}_{\boldsymbol{d}_{\boldsymbol{k}}}$ is the distribution of the process $\boldsymbol{d}_{\boldsymbol{k}}$, which includes all variables $d_{k-s}, s=0,1, \ldots$.

In this section, we head for an approximate solution to (5), which relies on the randomization of the probabilistic constraint according to the so called scenario approach, [15], [16], [14], [13], and the approximation of the stationary process $\boldsymbol{x}_{\infty}$ via its truncated counterpart $\boldsymbol{x}_{M}$.

These two approximations combined together lead to a computationally tractable convex optimization problem reformulation, with a solution that can be proven to be feasible for the original chance constrained optimization problem (5), with high probability.

Consider a set $\left\{d_{k-s}^{(i)}, s=0, \ldots, M\right\}_{i=1}^{N}$ of $N$ independent realizations of length $M+1$ of the disturbance process $\boldsymbol{d}_{\boldsymbol{k}}$ ("scenarios"). Then, we can formulate the following randomized and approximated version of (5):

$$
\begin{array}{rl}
\min _{\gamma \in \Gamma, \vartheta \in \Theta, h} & h \\
\text { subject to: } & \ell\left(x_{k, M}^{(i)}, \gamma+\vartheta d_{k}^{(i)}, d_{k}^{(i)}\right) \leq h-\delta \\
& f\left(x_{k, M}^{(i)}, \gamma+\vartheta d_{k}^{(i)}\right) \leq-\delta \\
& x_{k, M}^{(i)}=(I-A)^{-1} B \gamma+\sum_{s=0}^{M-1} A^{s}(B \vartheta+W) d_{k-1-s}^{(i)} \\
& i=1, \ldots, N
\end{array}
$$

where $\delta>0$ represents a tightening of the constraints that are probabilistically posed in (5).

Note that, under Assumption 4, the scenario optimization problem (6) is convex and can be efficiently solved via standard convex optimization techniques, [22]. In particular, when $\ell(\cdot)$ and $f(\cdot)$ are linear function of their first two arguments, and $\Gamma$ and $\Theta$ are box-sets, (6) is even an LP program.

We next introduce an assumption that is quite standard in scenario-based optimization (see, e.g., [14], [23]).

Assumption 6 (Feasibility): For any $N$, for any sample of disturbance realizations, the constrained optimization problem (6) is feasible and its feasibility set has a nonempty interior. Moreover, its solution exists and is unique.
Let $\left(\gamma^{\star}, \vartheta^{\star}, h^{\star}\right)$ be the optimal solution to (6). The following theorem provides guarantees about the feasibility of $\left(\gamma^{\star}, \vartheta^{\star}, h^{\star}\right)$ for the original problem (5).

Theorem 1 (Guarantees): Choose a confidence parameter $\beta \in(0,1)$ and suppose that $M \in \mathbb{N}$ and $\delta>0$ are chosen so that

$$
\tilde{\varepsilon}=\varepsilon-\frac{\mathcal{K}_{M}}{\delta}>0,
$$

where $\mathcal{K}_{M}>0$ is a given exponentially decaying to zero function of $M$ that is defined in [24]. Suppose also that the multi-sample size $N$ is chosen so as to satisfy ${ }^{1}$

$$
\sum_{i=0}^{n}\left(\begin{array}{c}
N \\
i
\end{array}\right) \tilde{\varepsilon}^{i}(1-\tilde{\varepsilon})^{N-i} \leq \beta,
$$

where $n$ is the number of scalar optimization variables in the controller parameters $(\gamma, \vartheta)$ in problems (5) and (6).

Then, if Assumptions 1-6 hold, the solution $\left(\gamma^{\star}, \vartheta^{\star}, h^{\star}\right)$ of the scenario program (6) is feasible for the original chance constrained problem (5) with probability larger than or equal to $1-\beta$, i.e.,

$$
\begin{aligned}
\mathbb{P}_{\boldsymbol{d}_{\boldsymbol{k}}}^{N}\left\{\mathbb { P } _ { \boldsymbol { d } _ { \boldsymbol { k } } } \left\{\ell\left(x_{k, \infty}^{\star}, \gamma^{\star}+\vartheta^{\star} d_{k}, d_{k}\right)\right.\right. & \leq h^{\star} \\
& \left.\left.\wedge f\left(x_{k, \infty}^{\star}, \gamma^{\star}+\vartheta^{\star} d_{k}\right) \leq 0\right\} \geq 1-\varepsilon\right\} \geq 1-\beta
\end{aligned}
$$

where $x_{k, \infty}^{\star}$ is the stationary process defined as the mean square limit of $x_{k, M}$ in (4) as $M \rightarrow \infty$, with $(\gamma, \vartheta)$ set equal to $\left(\gamma^{\star}, \vartheta^{\star}\right)$.

Proof: Consider the following optimization problem

$$
\min _{\gamma \in \Gamma, \vartheta \in \Theta, h} h
$$

subject to: $\mathbb{P}_{\boldsymbol{d}_{\boldsymbol{k}}}\left\{g\left(x_{k, M}, \gamma+\vartheta d_{k}, d_{k}, h\right) \leq-\delta\right\} \geq 1-\tilde{\varepsilon}$,

where $x_{k, M}$ is defined in (4), $\tilde{\varepsilon}$ is defined in (7), and

$$
g(x, u, d, h)=\max \{\ell(x, u, d)-h, f(x, u)\} .
$$

Since $\ell(x, u, d) \leq h$ and $f(x, u) \leq 0$ if and only if $g(x, u, d, h) \leq 0$, it follows from [14, Theorem 2.4] that the solution $\left(\gamma^{\star}, \vartheta^{\star}, h^{\star}\right)$ to the scenario program (6) with $N$ satisfying (8) is a feasible solution for the chance constrained problem (10) with probability at least $1-\beta$. Equivalently,

$\mathbb{P}_{\boldsymbol{d}_{\boldsymbol{k}}}^{N}\left\{\mathbb{P}_{\boldsymbol{d}_{\boldsymbol{k}}}\left\{g\left(x_{k, M}^{\star}, \gamma^{\star}+\vartheta^{\star} d_{k}, d_{k}, h^{\star}\right)>-\delta\right\} \leq \tilde{\varepsilon}\right\} \geq 1-\beta$,

where $x_{k, M}^{\star}$ is as in (4) with $(\gamma, \vartheta)=\left(\gamma^{\star}, \vartheta^{\star}\right)$.

Consider now the original chance constrained optimization problem (5), and notice that the left-hand side of its probabilistic constraint evaluated for $\left(\gamma^{\star}, \vartheta^{\star}, h^{\star}\right)$, namely, $\mathbb{P}_{\boldsymbol{d}_{\boldsymbol{k}}}\left\{g\left(x_{k, \infty}^{\star}, \gamma^{\star}+\vartheta^{\star} d_{k}, d_{k}, h^{\star}\right)>0\right\} \leq \varepsilon$, can be upper bounded as follows

$$
\begin{aligned}
\mathbb{P}_{\boldsymbol{d}_{\boldsymbol{k}}}\{ & \left.g\left(x_{k, \infty}^{\star}, \gamma^{\star}+\vartheta^{\star} d_{k}, d_{k}, h^{\star}\right)>0\right\} \\
& =\mathbb{P}_{\boldsymbol{d}_{\boldsymbol{k}}}\left\{g_{k, M}^{\star}+\delta+g_{k, \infty}^{\star}-g_{k, M}^{\star}-\delta>0\right\} \\
& \leq \mathbb{P}_{\boldsymbol{d}_{\boldsymbol{k}}}\left\{g_{k, M}^{\star}>-\delta \vee g_{k, \infty}^{\star}-g_{k, M}^{\star}>\delta\right\} \\
& \leq \mathbb{P}_{\boldsymbol{d}_{\boldsymbol{k}}}\left\{g_{k, M}^{\star}>-\delta\right\}+\mathbb{P}_{\boldsymbol{d}_{k}}\left\{g_{k, \infty}^{\star}-g_{k, M}^{\star}>\delta\right\},
\end{aligned}
$$

where we adopted the shorthand notations $g_{k, \infty}^{\star}=$ $g\left(x_{k, \infty}^{\star}, \gamma^{\star}+\vartheta^{\star} d_{k}, d_{k}, h^{\star}\right)$ and $g_{k, M}^{\star}=g\left(x_{k, M}^{\star}, \gamma^{\star}+\right.$ $\left.\vartheta^{\star} d_{k}, d_{k}, h^{\star}\right)$.

\footnotetext{
${ }^{1}$ See [25] for an explicit though slightly loose expression of $N$ as a function of $n, \tilde{\varepsilon}$, and $\beta$, satisfying (8).
} 
The second term in (13) can be bounded by means of the following chain of inequalities:

$$
\begin{gathered}
\mathbb{P}_{\boldsymbol{d}_{\boldsymbol{k}}}\left\{g_{k, \infty}^{\star}-g_{k, M}^{\star}>\delta\right\} \leq \mathbb{P}_{\boldsymbol{d}_{\boldsymbol{k}}}\left\{\left|g_{k, \infty}^{\star}-g_{k, M}^{\star}\right|>\delta\right\} \\
\leq \frac{\mathbb{E}_{\boldsymbol{d}_{\boldsymbol{k}}}\left[\left|g_{k, \infty}^{\star}-g_{k, M}^{\star}\right|\right]}{\delta} \leq \frac{\mathcal{K}_{M}}{\delta},
\end{gathered}
$$

where the second inequality is the Chebyshev's inequality and the proof of the last inequality is given in [24, Proposition 2] and not reported here due to space limitations. Combining (13) with (12) and (14), we obtain that

$$
\begin{aligned}
\mathbb{P}_{\boldsymbol{d}_{\boldsymbol{k}}}^{N} & \left\{\mathbb{P}_{\boldsymbol{d}_{\boldsymbol{k}}}\left\{g\left(x_{k, \infty}^{\star}, \gamma^{\star}+\vartheta^{\star} d_{k}, d_{k}, h^{\star}\right)>0\right\} \leq \tilde{\varepsilon}+\frac{\mathcal{K}_{M}}{\delta}\right\} \\
& \geq 1-\beta,
\end{aligned}
$$

which is (9) in Theorem 1, given the definition of $\tilde{\varepsilon}$ in (7) and the definition of $g$ in (11). This concludes the proof. Note that the solution $\left(\gamma^{\star}, \vartheta^{\star}, h^{\star}\right)$ to the scenario program (6) is a random quantity and, hence, the feasibility result in Theorem 1 holds with a certain probability $1-\beta$. Since the dependence of $N$ on $\beta$ is logarithmic, very small values of $\beta$ such as $10^{-6}$ or even $10^{-9}$ can be enforced without affecting $N$ too much, while providing a feasibility statement that, in practice, holds beyond any reasonable doubt.

Theorem 1 provides a similar result to the standard scenario theory. Indeed, in [14, Theorem 2.4] the same condition (8) on the multi-sample size is given except that $\varepsilon$ is used in place of $\tilde{\varepsilon}$, thus getting a lower value for $N$. We actually need to tighten the probability level from $\varepsilon$ to $\tilde{\varepsilon}$ by subtracting $\frac{\mathcal{K}_{M}}{\delta}$ from $\varepsilon$ to account for the approximation of $x_{k, \infty}$ by its truncated version $x_{k, M}$.

The presence of $\delta>0$ introduces some conservatism in two ways: i) tightening of the constraints, and ii) adoption of a smaller violation parameter $\tilde{\varepsilon}$ to determine the multi-sample size $N$. Decreasing $\delta$ reduces the conservatism in i) and increases that in ii). By different choices of $\delta$ we can tradeoff between i) and ii); none of them can be completely ruled out though.

The introduced conservatism can be effectively reduced by improving the quality of the approximation, which can be done at the expense of increasing the length $M$ of the disturbance realizations. Increasing $M$ mainly amounts of extracting longer disturbance realizations, which can be performed in most cases at low computational effort because neither the number of decision variables nor the number of constraints in problem (6) are affected. Therefore, by using large values of $M$, we allow to set $\delta$ close to zero and still get $\tilde{\varepsilon}$ close to $\varepsilon$.

\section{NUMERICAL EXAMPLE}

To show the efficacy of the proposed procedure we apply it to a realistic - though simplified - energy management problem. Consider a photovoltaic panel installation that is connected to the utility grid. Clearly, the amount of energy produced depends on the solar irradiation, which is unpredictable and can thus deviate significantly from a nominal production. Hence, a direct injection of the solar energy production to the grid may cause unpredictable fluctuations, and even abrupt changes in the network energy flow. To alleviate this issue, a battery is introduced to act as a buffer between the photovoltaic panel installation and the grid: in case of a mismatch between the actual and the nominal production profile, the battery can be charged/discharged to compensate.

Due to the daily periodicity of the solar irradiation and related energy production, the strategy to manage the battery is naturally designed by referring to a one-day time horizon. We next apply the approach presented in the paper to design a disturbance compensator off-line (and we need to do it only once!), with precise guarantees on its performance in the long run. More specifically, we are interested in managing the battery so as to minimize the maximum gap between the actual energy injected into the grid and a nominal profile within a one-day time frame. The latter will also be optimized and presented to the grid operator as a reference daily production profile together with deviation margins certified in probability.

To this end, we discretize the one-day time horizon in $T=$ 144 time slots of 10 minutes each. In each time slot $t \in \mathbb{N}$, the energy balance equation $E_{g}(t)=E_{p}(t)-E_{b}(t)$ must hold, where $E_{g}(t)$ is the energy exchanged with the grid, $E_{p}(t)$ is the solar energy production, and $E_{b}(t)$ is the amount of energy exchanged with the battery (i.e., $E_{b}(t)>0$ if the battery is charged and $E_{b}(t)<0$ if it is discharged). The battery is modeled as first order dynamical system described by the recursive equation $\xi(t+1)=a \xi(t)+E_{b}(t)$, where $\xi(t) \in \mathbb{R}$ represents the energy stored in the battery at the beginning of the time slot $t$, and $a=0.998$ is a coefficient taking into account self-discharging losses per time slot $t$.

We assume that the solar energy production $E_{p}(t)$ is a strictly cyclostationary process with period $T$, and known first and second order moments. Let $k$ be the day index and $d_{k}=$ $\left[E_{p}(k T) \cdots E_{p}((k+1) T-1)\right]^{\top}-\mu_{p}$, the daily solar energy production fluctuations with respect to the average profile $\mu_{p}=\left[\bar{E}_{p}(k T) \cdots \bar{E}_{p}((k+1) T-1)\right]^{\top}$. This entails that the introduced discrete time stochastic process $\boldsymbol{d}$ satisfies Assumption 2.

We can now write the day-by-day dynamics of the battery by first defining the battery energy content $x_{k}=\xi(k T)$ at the beginning of day $k$ and the battery charge/discharge commands during day $k u_{k}=\left[E_{b}(k T) \cdots E_{b}((k+1) T-1)\right]^{\top}$, and then rewriting the recursive equation for the battery as $x_{k+1}=A x_{k}+B u_{k}$, with $A=a^{T}$ and $B=\left[a^{T-1} \cdots a 1\right]$. Note that $A$ satisfies Assumption 1 since $a<1$.

The disturbance does not enter the system dynamics but it does enter the control input $u_{k}=\gamma+\vartheta d_{k}$, where $\gamma \in \mathbb{R}^{T}$ and $\vartheta \in \mathbb{R}^{T \times T}$. The goal of minimizing the maximum deviation of the actual energy exchanged with the grid $d_{k}+\mu_{p}-u_{k}$ (derived from the energy balance equation) during day $k$ from its nominal daily profile $\mu_{p}-\gamma$, is encoded by the following cost function

$$
\begin{aligned}
\ell\left(x_{k}, u_{k}, d_{k}\right) & =\left\|d_{k}+\mu_{p}-u_{k}-\left(\mu_{p}-\gamma\right)\right\|_{\infty} \\
& =\left\|(I-\vartheta) d_{k}\right\|_{\infty} .
\end{aligned}
$$

Also, constraints on the energy stored in the battery and on the energy exchanged with the battery per time slot $t$ are present. To guarantee the causality of the control action within the day, the amount of energy exchange with the battery $E_{b}(t)$ at time $t$ cannot depend on current and 
future values of the solar energy production $E_{p}(\tau), \tau \geq t$. Therefore, $\vartheta$ must have a strictly lower triangular structure, with zeros on the main diagonal. In order to keep low the number of optimization variables entering the bound in Theorem 1, only the first $r$ subdiagonals of $\vartheta$ are different from zero, with all elements on the same subdiagonal being equal, and the nominal compensation term $\gamma$ taken as a rescaled version of the nominal energy production profile $\mu_{p}$, i.e., $\gamma=c_{\gamma} \mu_{p}$ with $c_{\gamma} \in \mathbb{R}$.

The control design problem is then formulated as the following chance constrained optimization problem

$$
\min _{c_{\gamma} \in \Gamma, \vartheta \in \Theta, h} h
$$

subject to:

$$
\begin{aligned}
& \mathbb{P}_{\boldsymbol{d}_{\boldsymbol{k}}}\left\{\left\|(I-\vartheta) d_{k}\right\|_{\infty} \leq h,\left|c_{\gamma} \mu_{p}+\vartheta d_{k}\right| \leq s^{\max }\right. \\
& \left.\quad \xi^{\min } \leq A_{T} x_{k, \infty}+B_{T}\left(c_{\gamma} \mu_{p}+\vartheta d_{k}\right) \leq \xi^{\max }\right\} \geq 1-\varepsilon
\end{aligned}
$$

where $\xi^{\mathrm{min}}=3 \mathrm{MJ}$ and $\xi^{\max }=57 \mathrm{MJ}$ are the minimum and maximum values for the battery energy content (which we set to be equal to $5 \%$ and $95 \%$ of the battery total capacity of $60 \mathrm{MJ}), s^{\max }=1 \mathrm{MJ}$ is the maximum amount of energy that can be exchanged with the battery in one time slot, and $A_{T}$ and $B_{T}$ are suitable matrices of the unrolled battery dynamics such that $A_{T} \xi(k T)+B_{T}\left(c_{\gamma} \mu_{p}+\vartheta d_{k}\right)=$ $[\xi(k T) \cdots \xi((k+1) T)]^{\top}$.

As for the constant $\eta$ in [24, Proposition 1] that enters the expression of $\mathcal{K}_{M}$ in Theorem 1, we have that $\|B \vartheta+W\|_{1} \leq$ $\|B\|_{1}\|\vartheta\|_{1}=\|\vartheta\|_{1}$ because $W=0$ and $\|B\|_{1}=1$. If we then add $\|\vartheta\|_{1} \leq r$ as a further (convex) constraint to specify $\Theta, \eta$ can be finally upper bounded by $r$.

Notice that the evolution of the battery content $\xi(t), t=$ $k T+1, \ldots,(k+1) T$, within day $k$ is determined by its value $x_{k}$ at the beginning of the day and the control input $u_{k}$, which comprises the charge/discharge commands $E_{b}(t)$, $t=k T, \ldots,(k+1) T-1$. Since $u_{k}$ depends on the cyclostationary process $E_{p}(t)$, then, the joint stationarity of $\left(\boldsymbol{x}_{\infty}, \boldsymbol{d}\right)$ implies the cyclostationarity of $\xi(t)$ with daily period $T$. The constraints are linear in $x$ with Lipschitz constant $L=1$ and the disturbance $d_{k}$ is such that $\sigma=22.9971$.

Problem (15) fits the framework of (5), therefore it can be solved via the proposed methodology and comes with the guarantees provided by Theorem 1 . Setting $r=3$ the overall number of decision variable is $n=4$. To get a violation of $\varepsilon=0.1$ with a confidence level of $\beta=10^{-3}$ it suffices to follow these simple steps: i) set $\delta=10^{-2}$ so as not to overtighten the constraints; ii) use the expression of $\mathcal{K}_{M}$ to find $M=52$ so as to have, according to (7), $\tilde{\varepsilon}=0.0915$ close enough to $\varepsilon$; iii) use (8) to get $N=138$ via bisection. Solving the corresponding scenario program we get $c_{\gamma}^{\star}=$ 0.1473 and $\vartheta_{1}^{\star}=1.0084, \vartheta_{2}^{\star}=-0.6104, \vartheta_{3}^{\star}=0.1126$, and $h^{\star}=0.6253$. Thus, with confidence $1-\beta$, the probability that the actual energy exchange with the grid $E_{g}(t)$ belongs to the tube of width $2 h^{\star}$ centered on the nominal profile $\mu_{p}-\gamma^{\star}=\left(1-c_{\gamma}^{\star}\right) \mu_{p}$ for all $t$, is greater than $1-\varepsilon$.

To validate the approach we run $N_{v}=10^{4}$ simulations each one up to day $k=M$ starting from day $k=0$, using new realizations of the solar energy production disturbance (different from those adopted for the control policy design). In order to study the transient behavior of the system, we
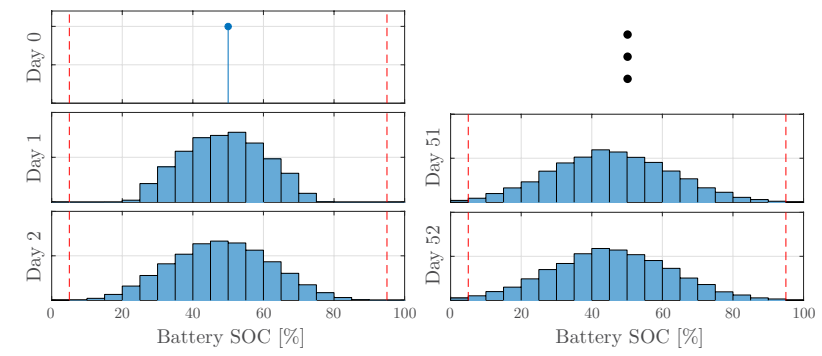

Fig. 1. Distribution of the battery SOC at day $k=0,1,2, M-1, M$ estimated based on $N_{v}$ simulations, with the SOC initialized at $50 \%$ in all of them.

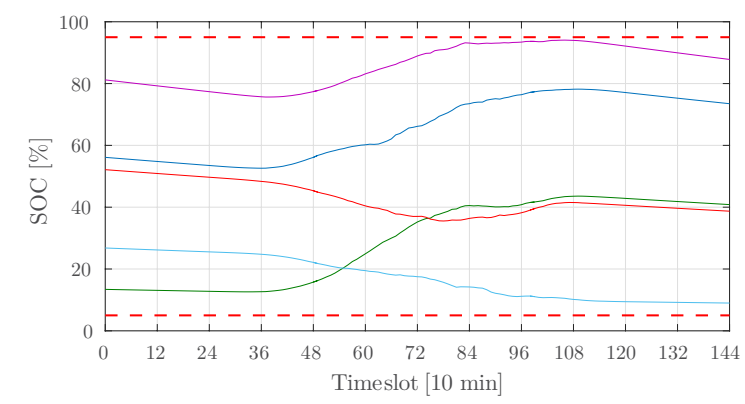

Fig. 2. Selected realizations of the battery SOC evolution within the $M$-th day. Dashed lines represents the threshold SOC values $5 \%$ and $95 \%$.

set the initial battery energy content equal to $30 \mathrm{MJ}$ ( $50 \%$ of its capacity) in all runs. For ease of exposition we plot the battery State Of Charge (SOC) in percentage.

In Figure 1 we can see the evolution of the histograms of the SOC as days progress. Plots refer to five days, the initial day $k=0$ and then those with $k=1,2, M-1, M$. As it can be seen from the picture, thanks to Assumptions 1 and 2, convergence to the stationary distribution is reached in about $M$ days (the histograms for days $M-1$ and $M$ are basically identical).

Figure 2 instead shows some possible realizations of the battery SOC profile within the $M$-th day. As can be seen, the designed compensator keeps the battery energy content within the prescribed limits, yet different qualitative behaviors can be observed that reflect the different possible realizations of the solar energy production disturbance $d_{k}$ within day $k=M$. The cyclostationarity of such a disturbance causes the distribution of $\xi(t)$ to be non-stationary within the day. To the purpose of showing the benefits of introducing the battery, we solved problem (15) setting $\gamma=0$ and $\vartheta=0$, which resulted in an optimal value for $h$ of $h_{w o}^{\star}=1.1615$, almost double with respect to the value $h^{\star}$ obtained operating the battery according to the optimal compensation policy. Note that, in order to have results with the same level of $\varepsilon$ for both cases, the number of scenarios $N$ to be extracted to compute $h_{w o}^{\star}$ is lower than 138. Moreover, since there is no battery, there is no stationary distribution to approximate and the standard scenario theory applies. In fact, the $N$ for the case without the battery can be obtained via bisection using (8) with $n=0$ and $\varepsilon$ in place of $\tilde{\varepsilon}$, which gives $N=66$. Moreover, in Figure 3, the reader can appreciate how the proposed approach is able to shape the distribution of the maximum deviation of the energy exchanged with the grid from its nominal daily profile, in order to squeeze its support 


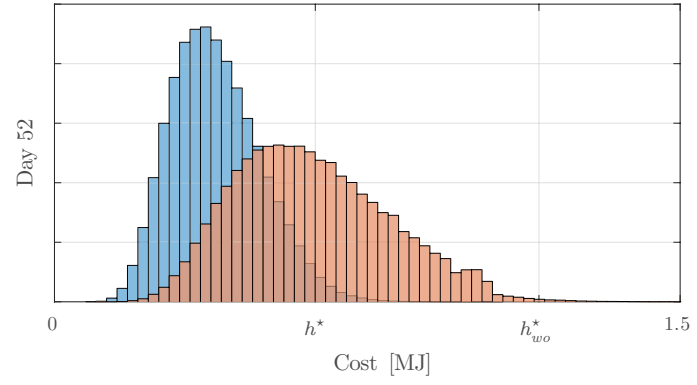

Fig. 3. Distribution of the maximum deviation of the energy exchanged with the grid from its nominal daily profile estimated by using $N_{v}$ realization of the solar energy production disturbance $d_{k}$, operating the battery with the optimal stationary policy (blue histogram) and without using the battery (orange histogram).

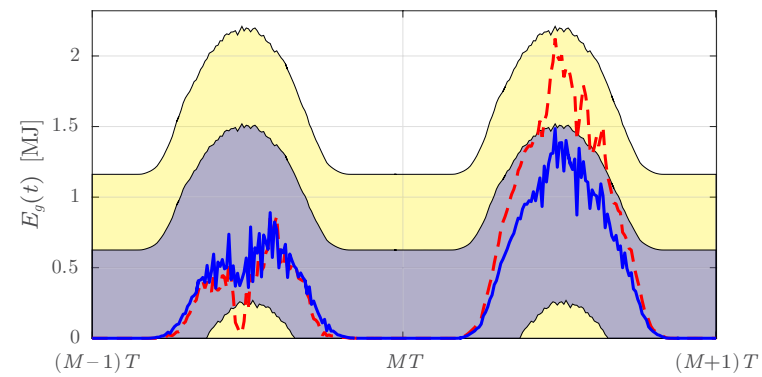

Fig. 4. Tubes of width $2 h^{\star}$ and $2 h_{w o}^{\star}$, centered in the nominal production profiles $\mu_{p}-\gamma^{\star}$ and $\mu_{p}$, for the cases with and without battery (blue narrow tube and orange wide, respectively), together with a two-day long realization of the energy exchanged with the main grid (solid blue line when the battery is present and optimally operated, and dashed red line with no battery).

towards zero.

Finally, in Figure 4, we report a two-day long realization of the energy exchanged with the main grid together with the tubes of width $2 h^{\star}$ and $2 h_{w o}^{\star}$, centered in the nominal production profiles $\mu_{p}-\gamma^{\star}$ and $\mu_{p}$, respectively, for the cases with (solid blue line) and without (dashed red line) battery.

\section{CONCLUSION AND FUTURE WORK}

In this paper, we address the design of a disturbance compensator for a discrete time linear system with non measurable state so as to optimize its performance in stationary regime while satisfying probabilistic joint state/input constraints. The proposed design strategy rests on the randomized solution to the chance constrained optimization program via the scenario approach, which is tailored to our framework in that only scenarios of a truncated version of the stationary state process of interest can be extracted. The effectiveness of the approach is illustrated on a simple energy management application example, where the designed compensator results in a periodic stochastic controller. We are currently working on the application of the approach to building energy management. We are also investigating the case when the disturbance is Gaussian, where the probability distribution of the stationary state admits an analytic expression and no truncation is hence needed.

\section{REFERENCES}

[1] K. Åström, Introduction to Stochastic Control Theory, ser. Mathematics in Science and Engineering. A Series of Monographs and Textbooks. Academic Press, 1970.
[2] D. Clarke and R. Hastings-James, "Design of digital controllers for randomly disturbed systems," Proc. IEE, vol. 118, no. 10, pp. 15031506, 1971.

[3] V. Peterka, "On steady state minimum variance control strategy," Kybernetika, vol. 8, no. 3, pp. 219-232, 1972.

[4] U. Shaked and P. Kumar, "Minimum variance control of discrete time multivariable armax systems," SIAM Journal on Control and Optimization, vol. 24, no. 3, pp. 396-411, 1986.

[5] M. Grimble, "Generalized minimum variance control law revisited," Optimal Control Applications and Methods, vol. 9, no. 1, pp. 63-77, 1988.

[6] B. Huang, "Minimum variance control and performance assessment of time-variant processes," Journal of Process Control, vol. 12, no. 6 , pp. 707-719, 2002.

[7] P. J. Gawthrop, "Minimum-variance control," in Encyclopedia of Life Support Systems (EOLSS), H. Unbehauen, Ed. UNESCO, 2004, ch. 6.43. Control Systems, Robotics and Automation. [Online]. Available: http://www.eolss.net

[8] H. Toivonen, "Minimum variance control of first-order systems with a constraint on the input amplitude," IEEE Transactions on Automatic Control, vol. 26, no. 2, pp. 556-558, Apr 1981.

[9] M. Grimble, "Controller performance benchmarking and tuning using generalised minimum variance control," Automatica, vol. 38 , no. 12 , pp. $2111-2119,2002$

[10] Z. Zhou and R. Cogill, "Reliable approximations of probabilityconstrained stochastic linear-quadratic control," Automatica, vol. 49, no. 8 , pp. $2435-2439,2013$.

[11] E. Cinquemani, M. Agarwal, D. Chatterjee, and J. Lygeros, "Convexity and convex approximations of discrete-time stochastic control problems with constraints," Automatica, vol. 47, no. 9, pp. 2082-2087, 2011

[12] D. Bertsimas and D. Brown, "Constrained stochastic LQC: A tractable approach," IEEE Transactions on Automatic Control, vol. 52, no. 10 , pp. 1826-1841, 2007.

[13] M. Campi, S. Garatti, and M. Prandini, "The scenario approach for systems and control design," Annual Reviews in Control, vol. 33, no. 2 , pp. $149-157,2009$.

[14] M. Campi and S. Garatti, "The exact feasibility of randomized solutions of uncertain convex programs," SIAM Journal on Optimization, vol. 19 , no. 3, pp. 1211-1230, 2008

[15] G. Calafiore and M. Campi, "Uncertain convex programs: randomized solutions and confidence levels," Mathematical Programming, vol. 102, no. 1, pp. 25-46, 2005.

[16] - "The scenario approach to robust control design," IEEE Transactions on Automatic Control, vol. 51, no. 5, pp. 742-753, 2006.

[17] D. Ioli, A. Falsone, and M. Prandini, "Optimal energy management of a building cooling system with thermal storage: a convex formulation," in Proceedings of the 9th IFAC Symposium on Advanced Control of Chemical Processes (ADCHEM 2015), Whistler, British Columbia, Canada, 2015.

[18] D. Ioli, A. Falsone, S. Schuler, and M. Prandini, "A compositional framework for energy management of a smart grid: a scalable stochastic hybrid model for cooling of a district network," in Proceedings of the 12th IEEE International Conference on Control and Automation (ICCA 2016), Kathmandu, Nepal, 2016.

[19] P. E. Caines, Linear stochastic systems. Wiley New York, 1988, vol. 11.

[20] A. Prèkopa, Stochastic programming. Boston, MT, USA: Kluwer, 1995.

[21] D. Dentcheva, "Optimization models with probabilistic constraints," in Probabilistic and Randomized Methods for Design under Uncertainty, G. Calafiore and F. Dabbene, Eds. London: Springer-Verlag, 2006.

[22] S. Boyd and L. Vandenberghe, Convex Optimization. Cambridge University Press, 2004.

[23] M. C. Campi and S. Garatti, "A sampling-and-discarding approach to chance-constrained optimization: Feasibility and optimality," Journal of Optimization Theory and Applications, vol. 148, no. 2, pp. 257-280, 2011.

[24] A. Falsone, L. Deori, D. Ioli, S. Garatti, and M. Prandini, "Supplement to the paper: Optimally shaping the stationary distribution of a constrained discrete time stochastic linear system via disturbance compensation," available on-line: http://home.deib.polimi.it/falsone/Supplement.pdf

[25] T. Alamo, R. Tempo, A. Luque, and D. R. Ramirez, "Randomized methods for design of uncertain systems: Sample complexity and sequential algorithms," Automatica, vol. 52, pp. 160 - 172, 2015. 